The difference between the results is too great to be accounted for by differences in care in scraping or errors of the weighings. It is either due to the fact that more dust had been carried off one table by the rain than off the other, or it shows that near the house the distribution was uneven. Still, the mean of the two gives an idea of the order of the density on the inner terrace, which was about 0.00I35 gram per square inch, or, taking $25 \mathrm{~mm}$. = I inch, 2.2 grams per square metre, or about $5 \frac{1}{2}$ tons per square mile, though, of course, to argue as to the average fall over so large an area as a square mile is a big extrapolation.

\section{Note upon a New Form of Spermatophore in an Earthworm.}

IT is well known that the Oligochæta (like many other animals such as molluscs, insects, \&c.) form spermatophores. Up to the present time two types of these spermatophores have been recognised in the Oligochæta. In the Lumbricidæ, Criodrilus, \&c., they are compact cases, generally open at one end, and found invariably attached to the outside of the body in the neighbourhood of the reproductive orifices; they are sometime even slightly imbedded in the skin. The other type of spermatophore characterises Tubrfex and several allied genera, as well as the Eudrilid earthworm, Polytoreutus. These are long thin motile bodies. They are found only in the spermathecæ of those Annelids which they characterise. I have lately studied the structure of a third variety of spermatophore which I found in the spermathecal sac of another Eudrilid genus, Stuhlmannia. This worm is long and slender, not measuring more than $2 \mathrm{~mm}$. in diameter. The spermathecal sac in which the spermatophores are found is about 7 or $8 \mathrm{~mm}$. long. I never found more than two of the spermatophores in a sac; they measure some $3 \mathrm{~mm}$. in length. The shape is peculiar. There is a "head" very similar to that of the spermatophore of Tubifex, a long "beak," open at the extremity, is followed by a circumferential swelling, which is again succeeded by a narrow neck; this in its turn gradually widens until at the middle the spermatophore is large and plump. The interior was a mass of separate spermatozoa entirely uncompacted together. The site of the formation of the spermatophores has been much disputed. It seems to be clear, from the investigations of Mr. Lankester, that in Tubifex they are at least moulded in the spermatheca, since the collar of the head of the spermatophore not only corresponded in form with certain foldings of the spermathecal wall, but was actually observed lying in situ. As to the origin of the material, $\mathrm{Mr}$. Lankester and Prof. Vejdovsky were disposed to trace it, partly at least, to the "cement gland" of the male efferent apparatus. I find in Stuhlmannia that the moulding of the head of the spermatophore must be accomplished in the spermathecal sac, since I also observed a spermatophore lying in situ with its convexities fitting into corresponding concavities of the walls of that sac. Further, the large size of the spermatophore necessitates the hypothesis that the whole of it is moulded in the sac, since it could not possibly be contained-even with the greatest stretching-in the spermiducal gland. As to the origin of the material of the case, I hold it to be proved that part comes from the spermiducal gland and that the rest is, in all probability, a result of the breaking down of the abundant cells which line the spermathecal sac. I may add that no spermatozoa protrude through the case. This spermatophore of Stuhlmannia is evidently somewhat intermediate in its characters between the two types already known.

Frank E. BEDdard,

Graphic Solutions of the Cubics and the Quartics.

ThE note by Prof. G. B. Mathews, F.R.S., published in NATURE of November I6, I899, has encouraged me to write the following, which may be also of some interest. I have considered not only the quartics, but also the cubics. For the quartics, the methods in this letter will be simpler than those of Prof. Mathews. We require to have a sheet of squared paper on which the curve $y=x^{3}$ for the cubic and the curve $y=x^{2}+x^{4}$ for the quartic have been printed.

I. For the cubic, take the curve $y=x^{3}$ and a straight line the equation of which is $\frac{x}{a}+\frac{y}{\mathrm{I}}=\mathrm{I}$. Then the abscissæe of the intersections of the curve and the straight line are the real roots of $x^{3}+-\mathrm{I}=0$. Now the general cubic is at once reducible to the form $z^{3}+p z+q=0$, and if we put $z=-x \sqrt[3]{q}$, this becomes $x^{3}+\frac{p}{q^{3}} x-\mathrm{I}=0$. This is identical with the former if $a=\frac{q^{3}}{p}$; so we can calculate $a$ very easily, then read off the real values of $x$, and finally take $z=-x \sqrt[3]{q}$.

II. For the quartic, take the curve $y=x^{2}+x^{4}$ and a straight line the equation of which is $\frac{x}{a}+\frac{y}{b}=\mathrm{I}$. Then the abscissæ of the intersections of the curve and the straight line are the real roots of $x^{4}+x^{2}+\frac{b}{a} x-b=0$. Now the general quartic is at once reducible to the form $z^{4}+p z^{2}+q z+r=0$, and if we put $z=x \sqrt{ } p$, this becomes $x^{4}+x^{2}+\frac{q}{p^{3}} x+\frac{r}{p^{2}}=0$. This is identical with the former if $a=-\frac{r}{q \sqrt{p}}, b=-\frac{r}{p^{2}}$; so we can calculate $a$ and $b$ very easily, then read off the real values of $x$, and finally take $z=x \sqrt{ } p$.

For the quartic, we can take also the parabola $y=x^{2}$ and a circle $(x-a)^{2}+y^{2}=\rho^{2}$. T. HAYASHr. Matsuyama Chūgakkō, Iyo, Japan, December 28, 1900.

"The Principles of Magnetism and Electricity."

THERE are two points occurring in the review (p. 434) of "The Principles of Magnetism and Electricity" in which the author appears to me to be correct, though the examples are given as instancing errors into which he has fallen. The reviewer states, "The author measures magnetic force in dynes and difference of potential in ergs," thereby apparently implying that this is incorrect. Surely these are usual units for expressing these quantities. Again, the author is taken to task for stating that in the case of a dynamo or motor armature, " owing to the self-induction of each section, a certain amount of energy is used twice in each revolution to establish the current in it. This energy is lost so far as the external circuit or the effective output of the machine is concerned." Whilst with a dynamo running sparklessly with copper brushes this is only partially true, the difficulty of obtaining the return of the energy thus absorbed is in practice so great that we see on a very large proportion of machines that carbon brushes are used, the object of which is to enable this energy to be wasted without an actual spark, and it is well known that machines with carbon brushes thus working have a higher rise of temperature from the waste of power than when true electrodynamic commutation takes place. Actual measurements of power alssorbed have also shown a waste of power from this cause sometimes exceeding 5 per cent. of the output of the machine.

London, March 12.

Llewelyn B. AtKinson.

THROUGH the courtesy of the Editor I am able to reply to the letter of Mr. Llewelyn B. Atkinson, in which he challenges two of my criticisms in the review of "The Principles of Magnetism and Electricity," by Mr. P. L. Gray. Mr. Atkinson states that I "apparently imply" that measuring magnetic force in dynes, and difference of potential in ergs, is incorrect. To prevent any misconception, I now wish to state quite definitely that I regard the above method of measurement as hopelessly and absolutely wrong. If it be correct, then magnetic force is a quantity whose dimensions are identical with those of dynamical force, and difference of potential a quantity of the dimensions of energy. If Mr. Atkinson is satisfied with these conclusions, and is prepared to uphold them, I have no further remarks to offer on the subject. I may, however, point out that measuring difference of potential in ergs is about as logical a proceeding as measuring difference of (gravitational) level in ft.-lbs. Some eccentric people might, no doubt, be found to uphold this latter proceeding. But a falsehood does not become a truth merely because a number of people give their unreasoning assent to it.

With regard to the second criticism, that relating to the process of commutation in a dynamo, I still maintain that the statement in Mr. P. L. Gray's book is misleading and incorrect in its generality, and $\mathrm{Mr}$. Atkinson practically admits as much in his letter. Nothing is said by Mr. Gray as to the particular statement in question being intended to apply to carbon brushes under certain conditions of use. It is a general statement, made without any qualifications, and as such is incorrect.

The REVIEWER. 